Gary L. Darmstadt, MD; Christopher P. Howson, PhD; Gijs Walraven, MD; Robert W. Armstrong, MD; Hannah K. Blencowe, MBChB; Arnold L. Christianson, FRCP Edin; Alastair Kent, MPhil; Helen Malherbe, MSc; Jeffrey C. Murray, MD; Carmencita D. Padilla, MD; Salimah R. Walani, PhD; for the Participant Working Group of the Dar es Salaam Seventh International Conference on Birth Defects and Disabilities in the Developing World

As the Sustainable Development Goals are adopted by United Nations member states, children with congenital disorders remain left behind in policies, programs, research, and funding. Although this finding was recognized by the creation and endorsement of the 63rd World Health Assembly Resolution in $\mathbf{2 0 1 0}$ calling on United Nations member states to strengthen prevention of congenital disorders and the improvement of care of those affected, there has been little to no action since then. The Sustainable Development Goals call for the global health and development community to focus first and foremost on the most vulnerable and those left behind in the Millennium Development Goal era. To maximize the opportunity for every woman and couple to have a healthy child and to reduce the mortality and severe disability associated with potentially avoidable congenital disorders and their consequences for the children affected, their families and communities, and national health care systems, we propose priority measures that should be taken urgently to address this issue.

JAMA Pediatr. 2016;170(8):790-793. doi:10.1001/jamapediatrics.2016.0388 Published online June 27, 2016.

\author{
Author Affiliations: Author \\ affiliations are listed at the end of this \\ article. \\ Group Information: The Participant \\ Working Group of the Dar es Salaam \\ Seventh International Conference on \\ Birth Defects and Disabilities in the \\ Developing World members are listed \\ at the end of this article. \\ Corresponding Author: Christopher \\ P. Howson, PhD, March of Dimes \\ Foundation, 1275 Mamaroneck Ave, \\ White Plains, NY 10605 \\ (chowson@marchofdimes.org)
}

$\mathrm{O}$ n September 21-24, 2015, the eve of the announcement of the Sustainable Development Goals (SDGs), stakeholders in adolescent, maternal, newborn, and child health and development from 37 countries convened in Dar es Salaam, Tanzania, for the Seventh International Conference on Birth Defects and Disabilities in the Developing World (ICBD) to discuss how to accelerate the prevention of congenital disorders (birth defects, ie, abnormalities of structure or function that are present from birth) and the improvement of care of affected children, especially in highburden, low-resource settings globally. ${ }^{1}$

This seventh conference, entitled Birth Defects in the PostMDG [Millennium Development Goals] Era: Joining Hands for Prevention and Care, was organized by March of Dimes in partnership with the Aga Khan Health Services-East Africa, the Aga Khan University-East Africa, US Centers for Disease Control and Prevention, and the Bill \& Melinda Gates Foundation. Since the first ICBD in 2001, each conference has been held in a different region of the developing world, with the goal of bringing together experts and stakeholders from the region and from around the world to discuss developments and highlight successes and issues to build capacity in lower-income countries for the prevention of birth defects and preterm birth and improvement of the care of those affected. The conference description, call for abstracts, key deadlines, and related materials were sent out periodically via email lists and the March of Dimes and Centers for Disease Control and Prevention listservs.
The ICBDs were established to focus attention on the need to improve the prevention and care of congenital disorders given that the proportion of deaths in children younger than 5 years due to congenital disorders is rising in many low- and middle-income countries. In addition, rates of disability resulting from congenital disorders are increasing in many countries where newborn survival is improving but quality health care is lagging. Congenital disorders and their associated disabilities result in a substantial emotional, social, and economic toll on affected individuals, their families, and the communities in which they live. The Dar es Salaam ICBD was assembled to agree on actions to reduce this toll, especially in vulnerable, marginalized populations. A draft consensus statement was presented and discussed by the audience on the last day of the conference, with major changes incorporated at that time. The final document was created by the authors and circulated to all conference participants, with 76 providing input and supporting the consensus statement. A working group was also established to explore additional ways to put this statement into action.

We support accelerating the prevention of congenital disorders and improvement of care of affected individuals, recognizing that:

- An estimated 7.9 million children are born each year with a genetic or partially genetic (multifactorial) congenital disorder, and several hundred thousand more are born with congenital disorders due to in utero insults after conception, such as infections, and exposure to teratogens, such as alcohol. ${ }^{2,3}$ 
- Of the 2.7 million newborns who die annually, more than 1 in 10 die of a congenital disorder, and, overall, there are an estimated 484000 deaths due to congenital disorders among children younger than 5 years. ${ }^{4}$ This number is likely a gross underestimate, however, because many deaths due to congenital disorders, such as heart defects and metabolic disorders, go undetected.

- It is likely that more than 192000 of the 2.6 million annual stillbirths may result from an underlying congenital disorder. ${ }^{5}$ The percentage of stillborn children affected by congenital disorders is likely much higher than for live births, and many efforts to prevent stillbirth will help reduce the occurrence of congenital disorders among stillborn children.

- Most newborns with a serious congenital disorder who survive face a lifetime of severe disability. ${ }^{2,3}$

- An estimated $94 \%$ of newborns with 1 or more congenital disorders are born in low- and middle-income countries, placing an additional severe burden on families, communities, and national health care systems. ${ }^{2,3}$

- Up to an estimated $70 \%$ of congenital disorders are preventable or their effect can be substantially mitigated and quality of life improved, but these preventive and mitigating actions are occurring almost exclusively in high-income settings. ${ }^{2,3}$

- The fact that children with congenital disorders have been left behind in policies, programs, research, and funding was recognized by the creation and endorsement of the 63rd World Health Assembly Resolution, ${ }^{6}$ calling in 2010 for United Nations member states to (1) raise awareness of congenital disorders as a cause of child morbidity and mortality; (2) develop and strengthen birth registration and surveillance for birth defects; (3) strengthen evidence on etiologic factors, diagnosis, and prevention of major birth defects; and (4) develop national plans for implementation of effective interventions to prevent and manage birth defects. This call by the World Health Organization, however, has gone unheeded except in pockets such as Southeast Asia, where a strategic framework ${ }^{7}$ is guiding efforts to prevent and control congenital disorders in 12 countries of the region.

The conference participants agreed that, with the advent of the SDGs, greater emphasis must be placed on more holistic approaches, including preventive care and, beyond survival, optimization of childrens' developmental potential. This emphasis aligns with the SDGs' call for equality in social inclusion and in opportunities for education, employment, and the ability for all human beings to fulfill their potential and enjoy prosperous, productive lives. Furthermore, the SDGs call for giving priority in policy and action to the most vulnerable, specifically including those with disabilities, and those currently most left behind. ${ }^{8}$ Congenital disorders can be considered the first chronic disease experienced in life and are encompassed in SDG goal 3 that calls for a reduction, by 2030, by onethird in premature mortality from noncommunicable diseases through prevention and treatment and the promotion of mental health and well-being. ${ }^{7}$

\section{Call to Action}

To maximize the opportunity for every woman and couple to have a healthy child; to reduce the consequences of potentially avoidable congenital disorders for those affected, their families, the health care system, and the wider society; and to promote the well-being of children who have a congenital disorder, there are many measures that should be taken urgently to address this issue. In this context and in order that no child is left behind, we pledge an initial focus that supports the following:

Improving data quality:

1. Building consensus on and widespread use of a standardized definition of congenital disorders, such as "abnormalities of structure or function, which are present from birth," ${ }^{(\mathrm{p} 2)}$ to facilitate data comparison and ensure that the contribution of congenital disorders to the burden of disease is comprehensively represented.

2. Establishing registries and surveillance systems and their integration, where possible, into existing data platforms to monitor the toll and risks of congenital disorders and evaluating the outcome of interventions for prevention and care. Consideration should also be given to the collection of pertinent data available from existing registries and surveillance systems in other countries.

Reducing risk:

1. Promoting family planning, allowing women and couples to choose when they have their first child, space their pregnancies, plan family size, define the ages at which they wish to complete their family, and reduce the proportion of unintended pregnancies.

2. Ensuring a healthy, balanced diet for girls and during a woman's reproductive years through an adequate intake of macronutrients (protein, carbohydrates, and fats) and a broad range of micronutrients. Special attention should be given to adding $400 \mu \mathrm{g}$ of synthetic folic acid daily to the diet through fortification and supplementation while also promoting a diet rich in food folates, correcting iodine deficiency through fortification, and ensuring iron sufficiency through fortification, supplementation, and therapy for those with deficiencies.

3. Removing teratogenic substances from the diet, the most important of which is alcohol, and minimizing environmental contaminants in foods.

4. Controlling infections in women of reproductive age, including rubella and syphilis, and optimizing maternal health through detection and management of chronic illnesses associated with an increased risk of congenital disorders, such as type 2 diabetes mellitus and epilepsy, which require teratogenic medications.

Improving care:

1. Training physicians, nurses, allied health care professionals, and workers in the fundamentals of the recognition, causes, and care of children with congenital disorders and ensuring physical examinations of all newborns by trained health care professionals before discharge from the hospital or clinic.

2. Aligning medical and social services to provide timely treatments for congenital disorders, including surgery, medications, dietary modifications, and rehabilitation services when needed.

3. Providing emotional and practical support for parents to enable them to understand and manage their risk of congenital disorders and to help families in supporting the growth and development of children with congenital disorders.

Empowering the public and civil society:

1. Educating the public about congenital disorders and the steps mothers and fathers can take with their health care professionals to maximize the chances of a healthy pregnancy. 
2. Strengthening civil society-including patient and parent support groups, faith-based groups, and nongovernmental organizations-to advocate for improved prevention of congenital disorders and access to high-quality, family-centered patient care, including facilitating community and professional awareness and education and advocating for increased funding for research on the causes of congenital disorders.

The following additional actions should be taken by countries as priorities and circumstances allow:

1. Training physicians, nurses, and allied health care professionals in the essentials of medical genetics. This training should include diagnosis of common congenital disorders before and at birth; means of treatment where possible in the primary health care setting; knowing when to refer a patient for more specialized treatment; basic genetic counseling, including best practices in communicatingunfavorable health information to parents; and support for families who have a child or are at risk of having a child with a congenital disorder. Genetic counseling aims to empower those who are counseled to make autonomous decisions regarding their health in ways that are consonant with their religious and ethical beliefs and circumstances and to support them in their decisions.

2. Establishing periconception medical services to assist women and their partners in attaining optimal physical and mental health and well-being and to facilitate a healthy pregnancy and delivery of a healthy infant. These services include rubella immunization; screening for the risk of genetic, partially genetic, and teratogenic congenital disorders; and mental health counseling, including identification and support for depression.

3. Implementing preconception or prenatal medical screening to identify women and couples at risk of having a baby with hemoglobin disorders; Down syndrome; blood type incompatibility; congenital sexually transmitted infections such as syphilis, human immunodeficiency virus, and herpes simplex virus; and structural malformations, particularly neural tube defects.
4. Establishing newborn screening to identify congenital hypothyroidism, phenylketonuria, galactosemia, sickle cell disease, glucose-6-phosphate dehydrogenase deficiency, and other metabolic disorders.

5. Supporting research into the diagnosis, prevention, etiologic factors, and treatment of congenital disorders to enable improved outcomes for children into the future.

The conference participants call for concerted action by international government policymakers and donor organizations to explore how these recommended actions can be funded through more cost-effective and rational integration of policy, funding, and interventions across the reproductive, maternal, newborn, child, and adolescent continuum. Systems are required that encourage more effective partnership among the many existing organizations and agencies whose missions address common risk factors and outcomes and who would benefit financially and programmatically from better integration of policy, research, and action at international and national levels.

\section{Conclusions}

The Seventh ICBD in Dar es Salaam, Tanzania, was a pivotal opportunity to build consensus and commitment for accelerated prevention of congenital disorders and improvement of care of affected children in low- and middle-income countries. In conjunction with the newly launched SDGs and building on the 63rd World Health Assembly resolution calling on United Nations member states to strengthen prevention and care for congenital disorders, immediate action on the plan outlined above will save newborn and child lives, reduce disability rates and improve quality of life in survivors, and substantially lessen the current emotional and economic toll of these conditions on affected individuals, their families, and the communities in which they live.

\section{ARTICLE INFORMATION}

Accepted for Publication: February 9, 2016.

Published Online: June 27, 2016.

doi:10.1001/jamapediatrics.2016.0388.

Author Affiliations: March of Dimes Prematurity Research Center, Department of Pediatrics, Stanford University School of Medicine, Stanford, California (Darmstadt); Department of Research and Global Programs, March of Dimes Foundation, White Plains, New York (Howson, Walani); Aga Khan Development Network, Geneva, Switzerland (Walraven); Department of Paediatrics, Faculty of Health Sciences, Aga Khan University, East Africa, Nairobi, Kenya (Armstrong); Centre for Maternal, Adolescent, Reproductive, and Child Health, London School of Hygiene and Tropical Medicine, London, United Kingdom (Blencowe); Wits Centre for Ethics, University of the Witwatersrand, Johannesburg, South Africa (Christianson); Genetic Alliance, London, United Kingdom (Kent); School of Clinical Medicine, College of Health Sciences, University of KwaZulu Natal, Durban, South Africa (Malherbe); Division of Global Health, Bill \& Melinda Gates Foundation, Seattle, Washington (Murray): Department of Pediatrics, College of Medicine, University of the Philippines, Manila (Padilla).

\begin{abstract}
Author Contributions: Drs Darmstadt and Howson had full access to all the data in the study and take responsibility for the integrity of the data and the accuracy of the data analysis.

Study concept and design: All authors.

Acquisition, analysis, or interpretation of data:

Darmstadt, Howson, Blencowe,

Drafting of the manuscript: Darmstadt, Howson,

Blencowe, Christianson, Kent, Malherbe, Padilla.

Critical revision of the manuscript for important

intellectual content: Darmstadt, Howson, Walraven,

Armstrong, Blencowe, Malherbe, Murray, Walani.

Statistical analysis: Howson.

Obtained funding: Murray.

Administrative, technical, or material support:

Darmstadt, Howson, Walraven, Armstrong,

Malherbe, Murray, Walani.

Study supervision: Darmstadt, Howson,

Christianson.
\end{abstract}

Conflict of Interest Disclosures: None reported.

Funding/Support: The Seventh International Conference on Birth Defects and Disabilities in the Developing World was supported in part by a grant from the March of Dimes Foundation, grant number 5U380T000199 from the US Centers for Disease Control and Prevention, and the Bill \& Melinda Gates Foundation (Investment ID OPP1129127).
Role of the Funder/Sponsor: The funding sources had no role in the design and conduct of the study; collection, management, analysis, and interpretation of the data; preparation, review, or approval of the manuscript; and decision to submit the manuscript for publication.

Group Information: The Participant Working Group of the Dar es Salaam Seventh International Conference on Birth Defects and Disabilities in the Developing World members include Kjersti Aagaard (Baylor College of Medicine); Neelam Aggarwal (Postgraduate Institute of Medical Education and Research, Chandigarh, India); Sujeewa Amarasena (Sri Lanka College of Paediatricians, Sri Lanka); Linda Barlow-Mosha (Makerere University, Johns Hopkins University Research Collaboration, Uganda); Muneerah Amin Vastani (Aga Khan University, School of Nursing and Midwifery, Tanzania); Eric Aswani Nyaligu (Technomed Limited, Nairobi, Kenya, and Perkin Elmer Distributors, Kenya); Tayo Awoyale, MD (Institute of Child Health and Primary Care, Lagos University Teaching Hospital, Lagos, Nigeria); Lieven Bauwens (International Federation for Spina Bifida and Hydrocephalus, Belgium); Martha Carvalho (Brazilian Genetic Alliance, Brazil); Harish Chellani (Department of Pediatrics, Safdarjang Hospital and Vardhman Mahavir Medical College, India); George 
Cosmas (Ministry of Health and Social Welfare, Tanzania); Sergio D'Agostino (Surgery for Children, Humanitarian Association, Vicenza, Italy); Matthew W. Darlison (World Health Organization Collaborating Centre for Community Control of Hereditary Disorders, University College London Centre for Health Informatics and Multi-professional Education, UK); Safiyya Devraj (Aga Khan Health Services, Tanzania); Rym El Rafei (American University of Beirut Medical Center, Lebanon); Munda Elias (Kakonko District Council, Tanzania); Martina Ens-Dokkum (Leiden University Medical Center, Leiden, the Netherlands); Chinyere V. Ezeaka (Neonatology Unit, Lagos University Teaching Hospital, Surulere, Lagos, Nigeria); Itziar Familiar-Lopez (Michigan State University); Lord Jephthah Joojo Gowans (The Ghana Cleft Research Project, Kwame Nkrumah University of Science and Technology, Ghana); Abby Grant (Medical Genetics Department, University Hospital of Northern Norway, Norway); Shobhna Gupta (Vardhman Mahavir Medical College and Safdarjung Hospital, New Delhi, India); Petri Huhtinen (Perkin Elmer, Africa, Finland); Fajolu Iretiola (College of Medicine, University of Lagos, Lagos University Teaching Hospital, Lagos, Nigeria); Kapila Jayaratne (Family Health Bureau, Ministry of Health, Sri Lanka); Alix Joseph (Perkin Elmer, France); Vijaya Kancherla (Emory University Rollins School of Public Health); Ajay Khera (Ministry of Health and Family Welfare, India); Jill Levy-Fisch (Save Babies Through Screening Foundation, USA); Desheng Liang (State Key Laboratory of Medical Genetics, Central South University, Changsha, China); Michele Lloyd-Puryear (Save Babies Through Screening Foundation and Parent Project Muscular Dystrophy, USA); William Macharia (Aga Khan University, Nairobi, Kenya); Luis Madeira (Ministry of Health, Mozambique); Jerusalem Makotore Muburirwa (Aga Khan Hospital, Dar es Salaam, Tanzania); Leonard James Malasa (Hubert Kairuki Memorial University, Dar es Salaam, Tanzania); Paola Manduca (University of Genoa and New Weapons Research Group, Italy); Janet Manoni (Friends of Children with Cancer, Tanzania); Shaba Michael Kilasi (Ministry of Health and Social Welfare, Tanzania); Franscesca Miquel-Verges (University of Arkansas for Medical Sciences); Shaffiq Mohamed (Aga Khan Hospital, Dar es Salaam, Tanzania); Lillian Mtei (Muhimbili University of Health and Allied Sciences, Tanzania); Edina Mullumba (Aga Khan
Health Service, Tanzania); Beata Mushema (Hubert Kairuki Memorial University, Tanzania); Rachel Musoke (University of Nairobi, Kenya); Wendy Nembhard (Arkansas Reproductive Health Monitoring System and University of Arkansas for Medical Sciences, College of Medicine, Department of Pediatrics, and Arkansas Children's Hospital Research Institute and Arkansas Center for Birth Defects Research and Prevention); Margaret Nguhi (Maternal, Neonatal and Child Health, Kenya); Irmgard Nippert (Westfaelische Wilhelms-Universitaet Muenster, Germany); Esther Nyambura Njoroge (Smile Train, USA); John Nelson Obondo (Buguruni Anglican Hospital, Tanzania); Comrade Lawal-Aiyedun Olubunmi (Spina Bifida and Hydrocephalus Care Foundation, Nigeria); Rebecca Opetsi Alitsi (Wezesha Kupaa: Youth With a Mission, Tanzania); Helena Pachón (Food Fortification Initiative, USA); Ysbrand Poortman (Preparing for Life Collaboration, the Netherlands); Charles Powell (Children's Hospital, Zinga, Tanzania); Lynne Powell (Rockford Memorial Hospital, USA); Neena Raina (Maternal, Newborn, Child and Adolescent Health, World Health Organization, Southeast Asia Regional Office, India); Rajarajeswari K (Sri Ramachandra University, India); Nagy Sabet (Misr University for Science and Technology, Egypt); Henry Safori (Ghana Health Service, Ghana); Rogath Saika Kishimba (Ministry of Health and Social Welfare, Field Epidemiology and Laboratory Training Program, Tanzania); Abdulhakim Salim Bayakub (The Registered Trustees of ASSAS Trust, Dar es Salaam, Tanzania); Hilal Salum (Comprehensive Community Based Rehabilitation in Tanzania, Tanzania); Florence Salvatory (Hubert Kairuki Memorial University, Tanzania); Switbert Sambala (Aga Khan Hospital, Dar es Salaam, Tanzania); Maria Teresa Vieira Sanseverino (Medical Genetics Service, Hospital De Clinicas De Porto Alegre, Brazil); Vivian Saria Frank (Kilimanjaro Christian Medical College, Tanzania); Iran Sayed-Raeisy (Aga Khan Health Services, Dar es Salaam, Tanzania); Neema Seya (MyRight-Empowers People with Disabilities, Tanzania); Hamisi Kimaro Shabani (Muhimbili Orthopedic Institute Department of Neurological Surgery, Tanzania); Alice Shalua (Bugando School of Nursing, Tanzania); Bradford Therrell (US National Newborn Screening and Global Resource Center, Texas); John Tole (Aga Khan University, Nairobi, Kenya); Christine Tusiime (CoRSU Rehabilitation
Hospital, Uganda); W. K. N. Weliwagamage (Family Health Bureau, Sri Lanka); D. L. Woods (Perinatal Education Trust, South Africa); and Lingqian Wu (Center of Prenatal Diagnosis, Xiangya Hospital Central South University, Changsha, China).

Additional Contributions: Megan Bruno and Randi Roberts, March of Dimes Foundation, provided administrative support in the preparation of the manuscript. They were not compensated for their contribution.

\section{REFERENCES}

1. March of Dimes. 7th International Conference on Birth Defects in the Developing World. http://icbd .marchofdimes.org/. Accessed October 1, 2015.

2. Christianson A, Howson C, Modell B. March of Dimes Global Report on Birth Defects: The Hidden Toll of Dying and Disabled Children. White Plains, NY: March of Dimes Birth Defects Foundation; 2006.

3. March of Dimes, World Health Organization. Management of Birth Defects and Haemoglobin Disorders: Report of a Joint WHO-March of Dimes Meeting. Geneva, Switzerland: World Health Organization; 2006.

4. Liu L, Oza S, Hogan D, et al. Global, regional, and national causes of child mortality in 2000-13, with projections to inform post-2015 priorities: an updated systematic analysis. Lancet. 2015;385 (9966):430-440.

5. Lawn JE, Blencowe $\mathrm{H}$, Waiswa $\mathrm{P}$, et al; Lancet Ending Preventable Stillbirths Series Study Group Lancet Stillbirth Epidemiology Investigator Group. Stillbirths: rates, risk factors, and acceleration towards 2030. Lancet. 2016;387(10018):587-603.

6. World Health Organization. Birth defects. http: //apps.who.int/gb/ebwha/pdf_files/WHA63/A63 _R17-en.pdf. Published May 21, 2010. Accessed October 1, 2015.

7. World Health Organization. Prevention and Control of Birth Defects in South-East Asia Region: Strategic Framework (2013-2017). New Delhi, India: World Health Organization; 2013.

8. United Nations Department of Economic and Social Affairs. Sustainable development topics. https://sustainabledevelopment.un.org/topics. Accessed October 1, 2015. 